\title{
Identification and characterization of regulatory pathways involved in early flowering in the new leaves of alfalfa (Medicago sativa L.) by transcriptome analysis
}

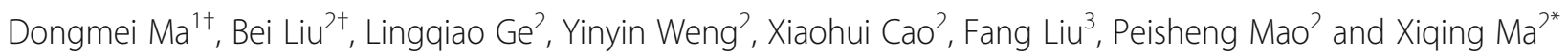

\begin{abstract}
Background: Alfalfa (Medicago sativa L.) is a perennial legume extensively planted throughout the world as a high nutritive value livestock forage. Flowering time is an important agronomic trait that contributes to the production of alfalfa hay and seeds. However, the underlying molecular mechanisms of flowering time regulation in alfalfa are not well understood.

Results: In this study, an early-flowering alfalfa genotype 80 and a late-flowering alfalfa genotype 195 were characterized for the flowering phenotype. Our analysis revealed that the lower jasmonate (JA) content in new leaves and the downregulation of JA biosynthetic genes (i.e. lipoxygenase, the 12-oxophytodienoate reductase-like protein, and salicylic acid carboxyl methyltransferase) may play essential roles in the early-flowering phenotype of genotype 80. Further research indicated that genes encode pathogenesis-related proteins [e.g. leucine rich repeat (LRR) family proteins, receptor-like proteins, and toll-interleukin-like receptor (TIR)-nucleotide-binding site (NBS)-LRR class proteins] and members of the signaling receptor kinase family [LRR proteins, kinases domain of unknown function 26 (DUF26) and wheat leucine-rich repeat receptor-like kinase10 (LRK10)-like kinases] are related to early flowering in alfalfa. Additionally, those involved in secondary metabolism (2-oxoglutarate/Fe (II)-dependent dioxygenases and UDP-glycosyltransferase) and the proteasome degradation pathway [really interesting new gene (RING)/U-box superfamily proteins and F-box family proteins] are also related to early flowering in alfalfa.

Conclusions: Integrated phenotypical, physiological, and transcriptomic analyses demonstrate that hormone biosynthesis and signaling pathways, pathogenesis-related genes, signaling receptor kinase family genes, secondary metabolism genes, and proteasome degradation pathway genes are responsible for the early flowering phenotype in alfalfa. This will provide new insights into future studies of flowering time in alfalfa and inform genetic improvement strategies for optimizing this important trait.
\end{abstract}

Keywords: Alfalfa, Flowering time, New leaves, Hormone, Transcriptome

\footnotetext{
*Correspondence: ma2016@cau.edu.cn

${ }^{\dagger}$ Dong Mei Ma and Bei Liu contributed equally to this work.

${ }^{2}$ College of Grassland Science and Technology, China Agricultural University, Beijing 100193, China

Full list of author information is available at the end of the article
}

(c) The Author(s). 2021 Open Access This article is licensed under a Creative Commons Attribution 4.0 International License, which permits use, sharing, adaptation, distribution and reproduction in any medium or format, as long as you give appropriate credit to the original author(s) and the source, provide a link to the Creative Commons licence, and indicate if changes were made. The images or other third party material in this article are included in the article's Creative Commons licence, unless indicated otherwise in a credit line to the material. If material is not included in the article's Creative Commons licence and your intended use is not permitted by statutory regulation or exceeds the permitted use, you will need to obtain permission directly from the copyright holder. To view a copy of this licence, visit http://creativecommons.org/licenses/by/4.0/ The Creative Commons Public Domain Dedication waiver (http://creativecommons.org/publicdomain/zero/1.0/) applies to the data made available in this article, unless otherwise stated in a credit line to the data. 


\section{Background}

Alfalfa (Medicago sativa L.) is a perennial leguminous forage extensively planted as livestock feed around the world due to the high crude protein and nutrition contents in leaves $[1,2]$. While flowering time is an important determinant of harvesting time, which contributes to forage quality, silage, yield, and production in alfalfa [3]. Early flowering can improve the number of cuttings, forage yield, and year-round production of alfalfa; it can also help to avoid the rainy season or drought stress for seed production, and enhance winter hardiness related to the temperate semi-arid continental climate in China [4]. Despite the importance, the underlying molecular mechanisms involved in flowering time control in alfalfa remain elusive.

Past endeavors of flowering time studies discovered six major regulatory pathways in model plants Arabidopsis, rice (Oryza.sativa L.), wheat (Triticum aestivum L.), and barley (Hordeum vulgare L.) [5, 6]. These pathways can be categorized into two groups: the photoperiod, vernalization, and ambient pathways that respond to environmental conditions, and the autonomous, gibberellin, and age pathways that respond to endogenous cues associated with the up/downregulation of floral integrator genes, including FLOWERING LOCUS $T$ (FT), FLOWERING LOCUS C (FLC), CONSTANS (CO) and SUPPRESSOR OF OVEREXPRESSION OF CONSTANS1 (SOC1) [7-9]. Furthermore, small non-coding RNAs and epigenetic pathways play important roles in regulating flowering time in plants [10]. For instance, in the plant adult phase, miR156 targets SQUAMOSA PROMOTER BINDING-LIKE (SPL) and induces flowering by upregulation expression of flower-promoting genes in the shoot apex [11], and FLOWERING LOCUS D, histone demethylase, downregulates FLC expression associated with mediating H3K4 demethylation and therefore promotes flowering in Arabidopsis [12]. An earlier study also indicated that jasmonate-activated (JA) transcription factors $M Y C 2, M Y C 3$, and $M Y C 4$ negatively regulate flowering time by interacting with $F T$, and exogenous JA could delay flowering time in Arabidopsis [13]. Applying exogenous abscisic acid (ABA) or inducing the overexpression of ABI5, an ABSCISIC ACID-INSENSITIVE MUTANT 5, through FLC expression can also trigger delayed flowering in Arabidopsis [14, 15]. In addition, receptor-like kinase (RLK) family proteins play a key role in the perception of internal and external signals involved in flowering time regulation [16]. For instance, FLOR1, a leucine-rich repeat (LRR) protein expressed in the shoot meristem, physically interacts with AGAMOUS (AG, a MADS-box transcription factor) and is induced in the early inflorescence meristem, and the Arabidopsis flor 1 mutant exhibits a delayed flowering phenotype [17]. CURVY1, a novel receptor-like protein kinase, also responds to flowering time and seed production in Arabidopsis [18]. Furthermore, the ubiquitinproteasome system is a major catabolic pathway involved in plant growth, development, and physiological processes, including floral development. Tagging of the target proteins with ubiquitin is mainly performed by E1 (ubiquitin-activating enzyme), E2 (ubiquitin-conjugating enzyme), and E3 (ubiquitin-protein ligase enzyme), followed by degradation mediated by the $26 \mathrm{~S}$ proteasome [19]. For example, PUB13, a U-box protein possessing E3 ubiquitin ligase, physically interacts with HFR1 (long hypocotyl in far-red light1) to regulate photomorphogenesis and flowering time in Arabidopsis [20]. However, whether their alfalfa homologs participate in flowering time regulation is not clear.

Previous studies using the shoot tip or leaf samples of alfalfa plants of different flowering stages have indicated that proteins involved in carbohydrate metabolism, phenylpropanoid biosynthesis and immunity are responsive to flowering time [4, 21]. However, little is known about the underlying molecular mechanisms associated with flowering time control in new leaves, which represent the transition from the vegetative to the floral phase in alfalfa. It has been shown that leaf development and floral organ initiation are controlled by common homeotic factors [22]. For instance, knocking down the $A G$ gene resulted in the reprogramming of leaf development and affected the expression of key regulatory genes (TRIPTYCHON, APETALA3 and PISTILLATA) in Arabidopsis leaves [22, 23]. Therefore, we hypothesize that these metabolic genes may play essential roles in new leaves of alfalfa during the early flowering stage. Here, integrated phenotypical, physiological, and transcriptome analyses showed that hormone biosynthesis and signaling pathway genes, pathogenesis-related (PR) genes, signaling receptor kinase family genes, secondary metabolism genes, and proteasome degradation pathway genes might contribute to the early flowering phenotype in alfalfa.

\section{Results}

Phenotypes and endogenous hormone contents

For the flowering time assays under controlled growth chamber conditions, plants were trimmed to maintain the same height. Genotype 80 first flowered at 25 days after planting with a mean plant height of $30.95 \mathrm{~cm}$, when the late-flowering genotype 195 had an average plant height of only $19.73 \mathrm{~cm}$ (Fig. 1a). Genotype 195 then flowered at 50 days after planting. Endogenous hormone contents in mature leaves (ML), new leaves (NL) including apical meristem, and flower buds (FB) were determined in both genotypes at 25 days after planting when genotype 80 flowered (Fig. $1 \mathrm{~b}$ and c; Fig. 2a-d). We observed significantly higher auxin (IAA), ABA, 


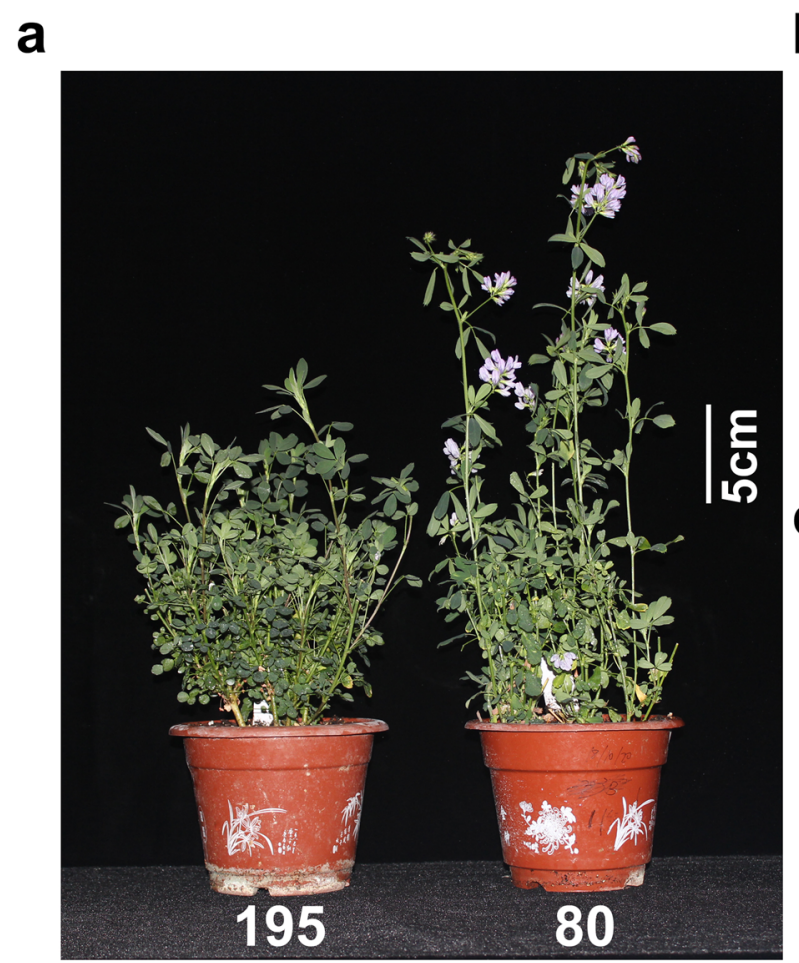

\section{b}
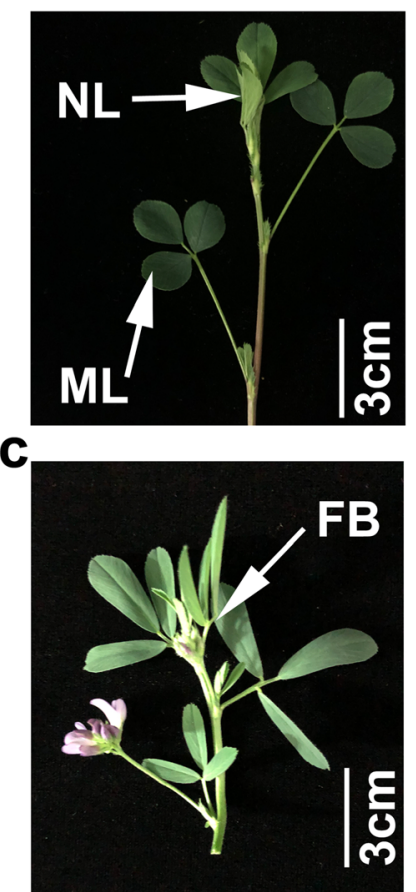

Fig. 1 Phenotypic characterization of genotypes 195 and 80 of alfalfa. a, The phenotype of genotype 195 and 80 plants grown in a controlled climate chamber at the time of sampling (25 days after plants). b and c, Tissues harvested for transcriptome analysis. ML, mature leaves; NL, new leaves including the apical meristem; FB, flower buds

salicylic acid (SA), and JA contents in NL compared with ML in both genotypes, while, the differences in hormone levels between NL and ML was smaller in genotype 80 than in 195 (Fig. 2a-d). In addition, except for IAA, hormone contents in FB were significantly higher than those in ML or NL in genotype 80 (Fig. 2a-d). Together, these results suggest a higher translocation efficiency of hormones from new leaves to flower buds in genotype 80 than genotype 195.

\section{Transcriptome profiling of leaves and flower buds}

To further investigate the molecular basis of the earlyflowering phenotype in genotype 80 , we performed transcriptome profiling in the ML, NL, and FB of both genotypes (Table 1). A total of $101.13 \mathrm{~Gb}$ of clean reads were obtained, the Q30 percentage (the percentage of bases with a quality score of 30 or higher) and GC (guanine and cytosine) percentage were 93.69 and $42.28 \%$, respectively. A total of 53,897 unigenes were yielded from the assembly (N50 of $1770 \mathrm{bp}$, mean length of $1106 \mathrm{bp}$ ) and were annotated using seven functional databases, including the NCBI non-redundant protein database (NR), Nucleotide sequence database (NT), Clusters of Orthologous Groups of proteins (COG), Gene Ontology (GO) and Kyoto Encyclopedia of Genes and Genomes (KEGG), Swissprot and Interpro (E-value $<10^{-5}$ ). Then,
31,962 coding sequence transcripts (N50 of $1866 \mathrm{bp}$, mean length of $1336 \mathrm{bp}$ ) were used for further functional analysis (Table 1). We identified 2148 (1454 upregulated/694 downregulated) and 1972 (1142 upregulated/ 830 downregulated) differentially expressed genes (DEGs) between NL and ML in 195 and genotype 80 respectively, among which 942 and 766 DEGs were exclusively expressed in genotype 195 and 80, respectively (Fig. 3a and b; Additional file 1: Table S1). In addition, 4423 (2300 upregulated/2123 downregulated) DEGs were identified between FB and NL in genotype 80, and 6288 (3615 upregulated/2673 downregulated) DEGs were identified between FB and ML in genotype 80, among which 2802 common DEGs were found to respond to early flowering in genotype 80 (Fig. $3 \mathrm{a}$ and b; Additional file 1: Table S1).

\section{Response of hormone-related genes to early flowering}

The significant change in hormone levels prompted us to further examine the transcript levels of hormonerelated genes. The analysis detected 27 and 34. DEGs (between NL and ML) involved in the IAA, ABA, SA and JA metabolism and signaling pathways in genotype 195 (NL195/ML195) and genotype 80 (NL80/ML80), respectively (Figs. 4a, b and 5; Additional file 2: Table S2). Among these DEGs, 11 of the 16 IAA metabolism and 


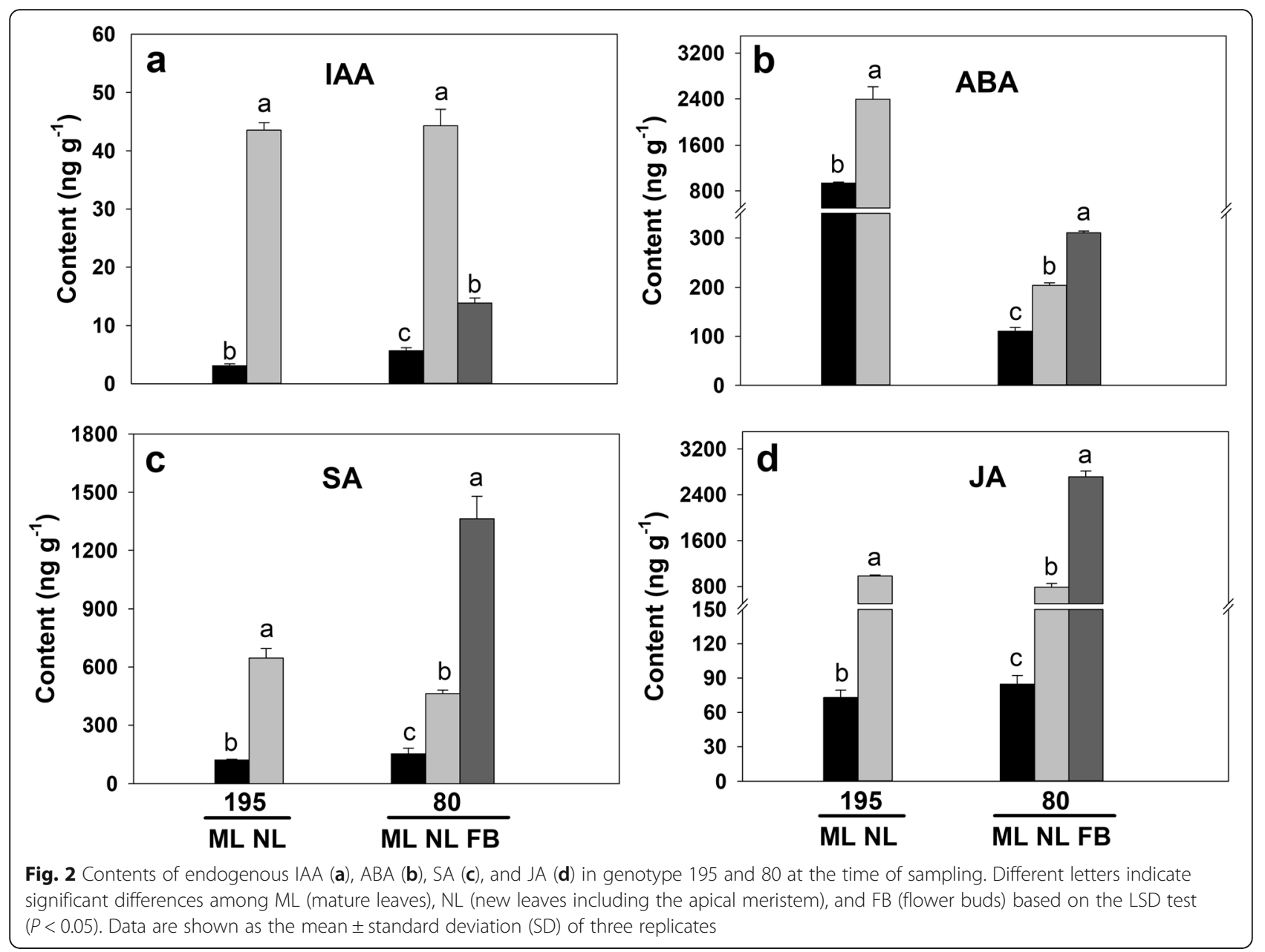

signaling pathway genes in NL were significantly downregulated compared with ML in genotype 80 (Fig. 5; Additional file 2: Table S2). Seven of the 13 IAA-related genes were downregulated in NL compared with ML in genotype 195, such as those encoding SAUR family proteins, o-fucosyltransferase, auxin efflux carrier component 5, and thromboxane-A synthase (Fig. 5; Additional file 2: Table S2). Furthermore, ten DEGs in genotype

Table 1 Summary of the transcriptome analysis of alfalfa leaves and flower buds

\begin{tabular}{ll}
\hline Total clean reads (Gb) & 101.13 \\
Q30 bases (\%) & 93.69 \\
GC content (\%) & 42.28 \\
Total number of unigenes & 53,897 \\
N50 of unigenes (bp) & 1770 \\
Mean length of unigenes (bp) & 1106 \\
Number of transcripts (coding sequence, CDS) & 31,962 \\
N50 of transcripts (bp) & 1866 \\
Mean length of transcripts (bp) & 1336 \\
\hline
\end{tabular}

195 (five downregulated/five upregulated) and eight DEGs in genotype 80 (three downregulated/five upregulated) were enriched in NL compared with ML (Fig. 5; Additional file 2: Table S2). These were primarily involved in ABA metabolism and signaling pathways, including the ABA-responsive protein, 9-cisepoxycarotenoid dioxygenase, and HVA22-like protein encoding-genes (Fig. 5; Additional file 2: Table S2). Two SA-related genes that encode SA carboxyl methyltransferase (SAMT) and lipoxygenase were significantly downregulated in NL compared with ML in genotype 80 but were not enriched in genotype 195 (Fig. 5; Additional file 2: Table S2). Seven of the nine JA biosynthetic genes were significantly downregulated in NL compared with ML in genotype 80; while two of the four JArelated genes were downregulated in NL compared with ML in genotype 195, including those encoding 12oxophytodienoate reductase-like protein, allene oxide synthase, and lipoxygenase (Fig. 5; Additional file 2: Table S2). These results further indicate that the downregulation of these hormone-related genes may have a negative impact on early flowering in genotype 80 . In 
a

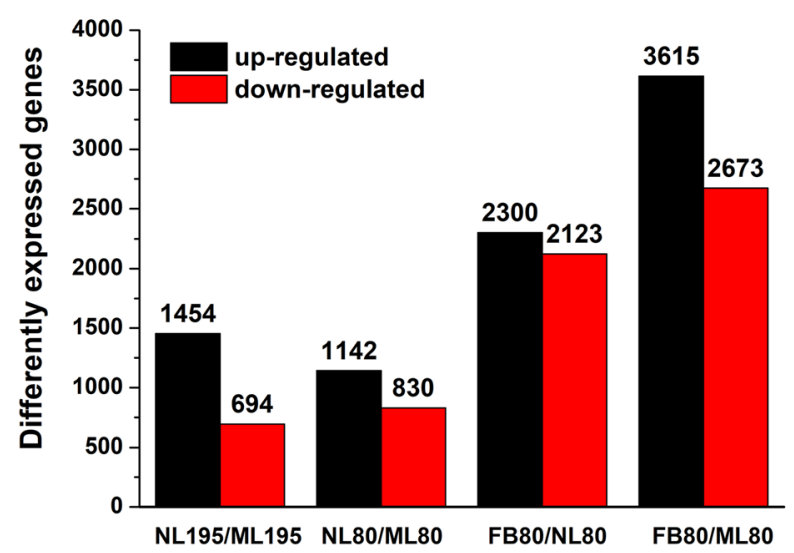

b

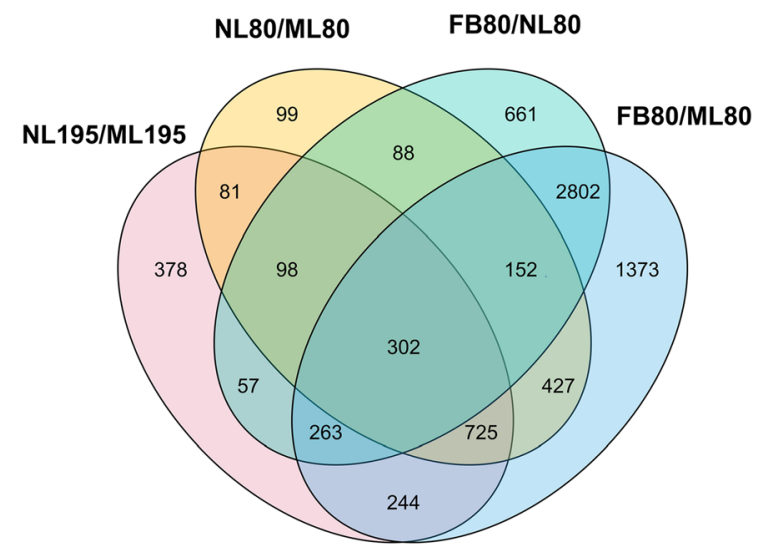

Fig. 3 The bar chart (a) and Venn diagram (b) of differentially expressed genes (DEGs) in genotypes 195 and 80

addition, 16 and 27 DEGs related to hormone metabolism and signaling pathways were identified between FB and $\mathrm{NL}$ and between FB and ML, respectively, in genotype 80; these include genes involved in ABA signal transduction and genes encoding auxin-responsive proteins, SAMT, allene oxide synthase, and lipoxygenase 3 (Fig. 5; Additional file 2: Table S2).

\section{Response of genes- related to pathogenesis and metabolism to early flowering}

Thirty-five DEGs (three upregulated/32 downregulated) in genotype 195 and 45 DEGs (one upregulated/44 downregulated) in genotype 80 were significantly enriched in NL compared with ML. These mainly include genes involved in the Toll-interleukin-like receptor (TIR)-nucleotidebinding site (NBS)-LRR and coiled-coil (CC)-NBS-LRR classes and those encoding LRR family proteins, NB-ARC (nucleotide-binding adaptor shared by APAF-1, R proteins, and CED-4) domain-containing proteins, and receptor-like proteins (Figs. 4a, b and 6; Additional file 2: Table S2). Meanwhile, 29 (three upregulated and 26 downregulated) DEGs between FB and NL and 57 (two upregulated and 55 downregulated) DEGs between FB and ML were identified in genotype 80 (Additional file 2: Table S2). We found that 66 metabolic DEGs between NL and ML (43 upregulated and 23 downregulated) were significantly enriched in genotype 195; whereas 82 such DEGs (47 upregulated and 35 downregulated) were enriched in genotype 80 . These mainly include genes encoding 2-oxoglutarate and Fe (II)-dependent oxygenase superfamily proteins (2OG oxygenases), chalcone synthase, laccases, and UDP-Glycosyltransferase superfamily proteins (UGTs) (Figs. 4a, b and 6; Additional file 2: Table S2). Fifty (40 upregulated and 10 downregulated) DEGs a

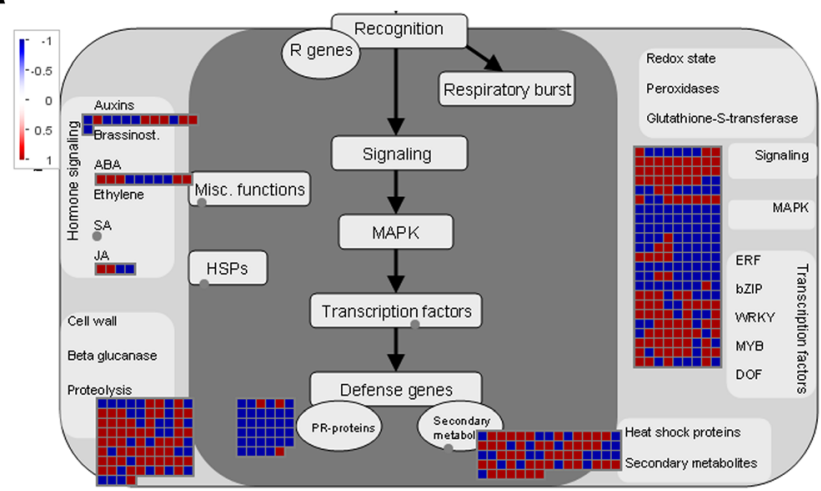

b

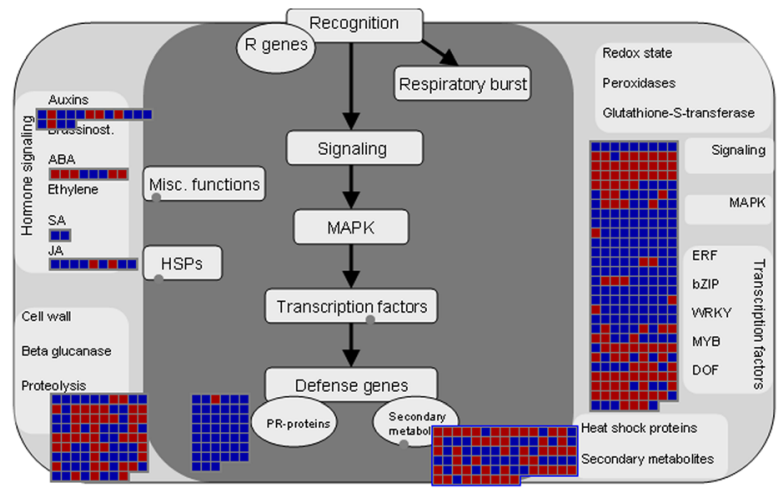

Fig. 4 MapMan display of the functional categories of DEGs between NL and ML in genotypes 195 (a) and 80 (b) upon the flowering of genotype 80. Squares represent DEGs; red and blue indicate up- and downregulated genes, respectively 


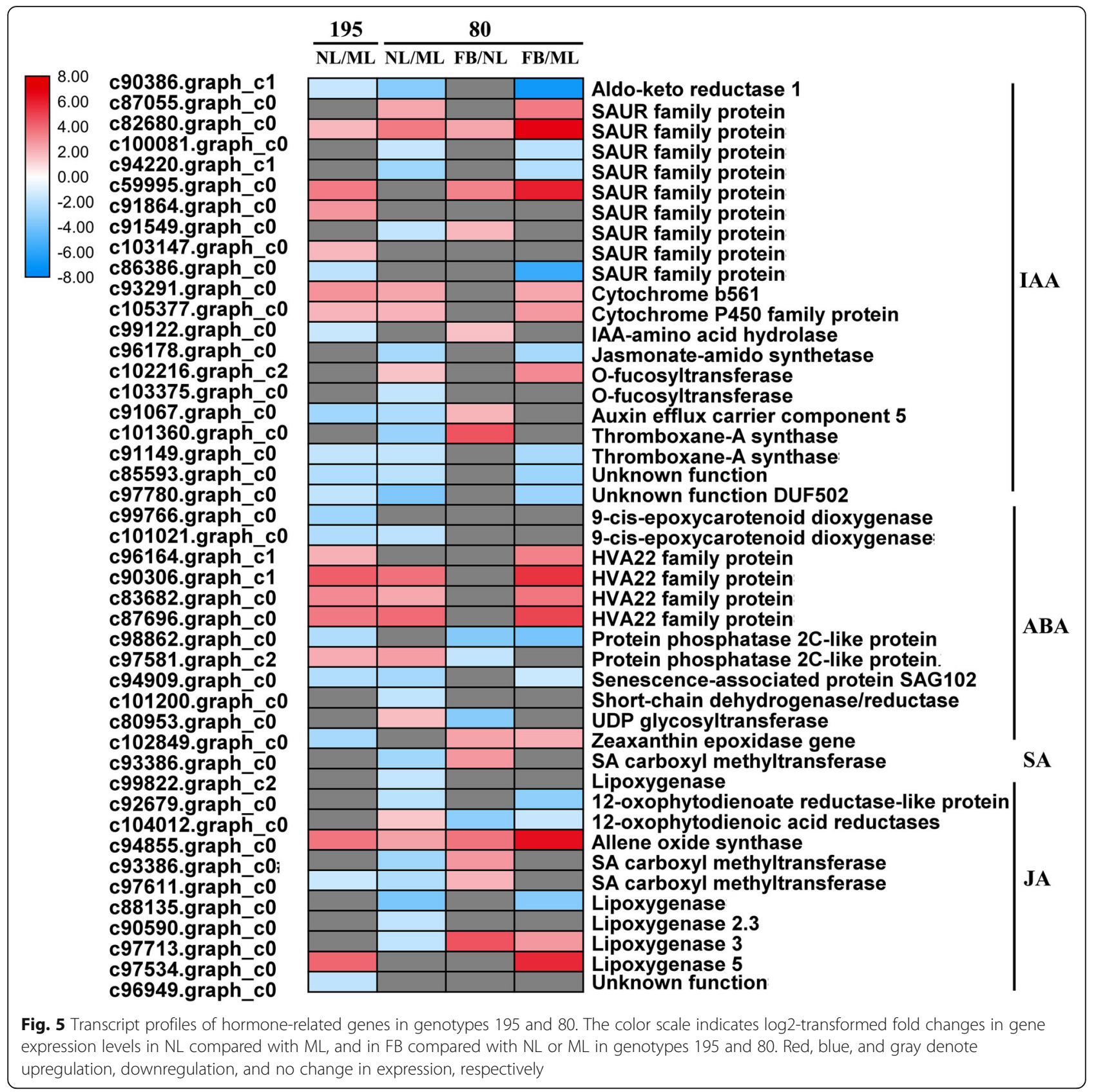

between FB and NL and 78 (50 upregulated and 28 downregulated) DEGs between $\mathrm{FB}$ and $\mathrm{ML}$ were identified in genotype 80 (Additional file 2: Table S2). Altogether, these findings indicate that compared with genotype 195, more PR genes were downregulated, and more metabolismrelated genes were upregulated in genotype 80 upon flowering.

Responses of receptor-like kinase family genes to early flowering

Furthermore, we observed differences in the expression of receptor-like kinase family genes in response to early flowering. For example, 117 DEGs between NL and ML (48 up-regulated and 69 down-regulated) were identified in genotype 195, and 162 such DEGs (52 up-regulated and 110 down-regulated) were identified in genotype 80 . These mainly include genes encoding cytoplasmatic kinases, LRR-kinases, the DUF 26 (domain of unknown function 26) receptor-like kinase, the wheat LRK10 (leucine-rich repeat receptor-like kinase10)-like receptor kinase, and S-locus glycoprotein-like kinases (Figs. 4a, b and 7; Additional file 2: Table S2). Meanwhile, 83 DEGs between FB and NL (24 up-regulated and 59 downregulated) and 177 DEGs between FB and ML (57 up- 


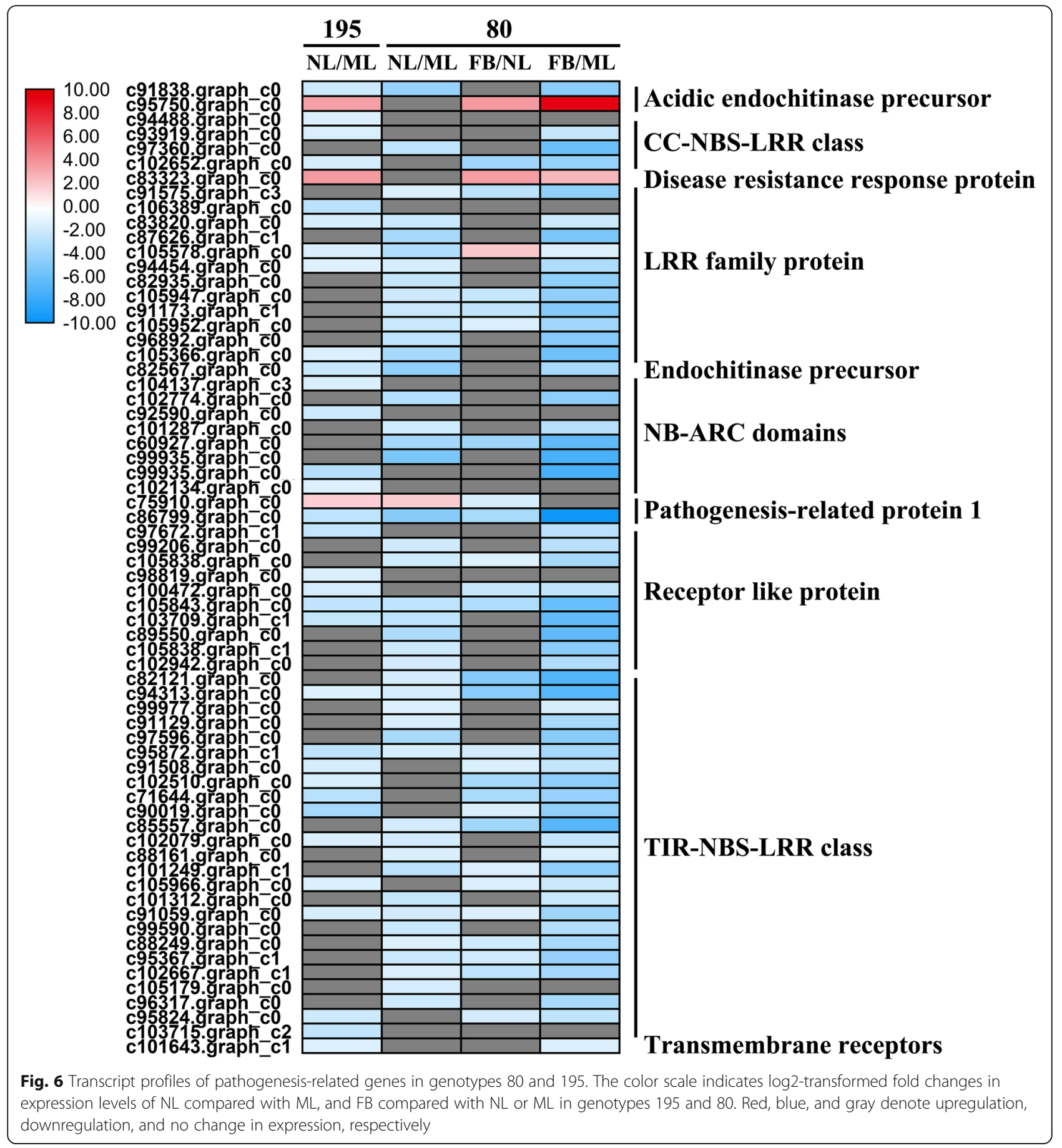

regulated and 120 down-regulated) were identified in genotype 80 (Additional file 2: Table S2). Taken together, these results reveal that more receptor-like kinase genes were downregulated in response to early flowering in genotype 80 than in genotype 195.

Response of proteasome-related genes to early flowering Thirty-two proteasome-related DEGs between NL and ML (21 up-regulated and 11 down-regulated) were identified in genotype 195, and 25 such DEGs (nine upregulated and 16 downregulated) DEGs were identified genotype 80 . These genes mainly encode F-box family proteins, really interesting new gene (RING) /U-box superfamily proteins, and S-ribonuclease binding proteins (Fig. 4a, b and 8; Additional file 2: Table S2). Meanwhile, ten DEGs between FB and NL (four upregulated and six down-regulated) and 32 DEGs between FB and ML (16 upregulated and 16 downregulated) were 


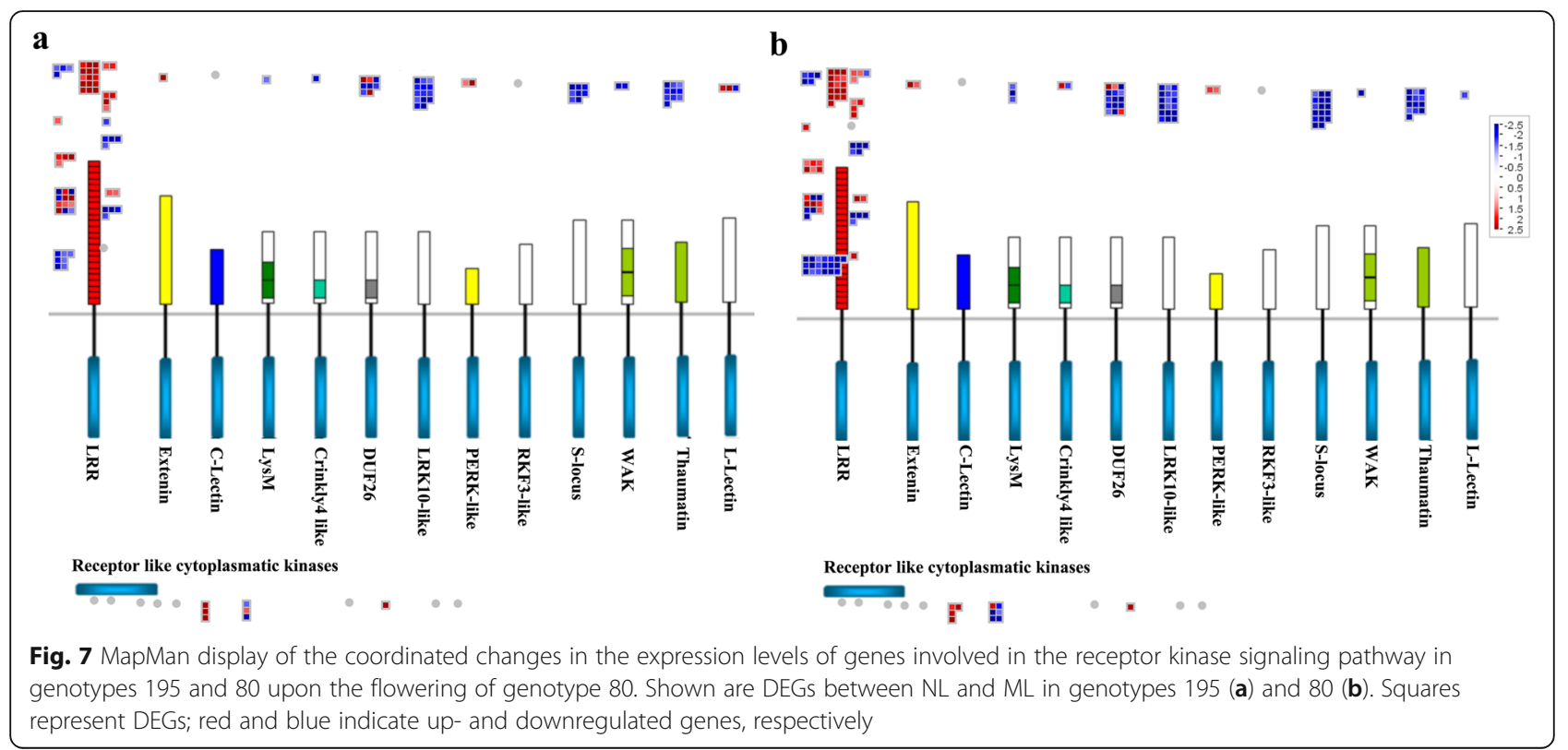

identified in genotype 80 (Figs. 8 and 9; Additional file 2: Table S2). Together, these results reveal that more proteasome related genes were downregulated in response to early flowering in genotype 80 .

\section{qRT-PCR validation of DEGs identified by RNA-seq}

We randomly selected 15 DEGs between NL and ML identified by transcriptome profiling from genotypes 195 for qRTPCT validation. Six of the 15 DEGs showed good agreement between the qRT-PCR analysis and the RNAseq data. These include genes involved in ABA signal transduction, and those encoding beta-amyrin synthase $\mathrm{R}$, a tyrosine kinase family protein, an LRR receptor-like kinase, and a RING/U-box superfamily protein. For genotype 80, the expression of all DEGs between NL and ML exhibited a high correlation between the RNAseq data and qRT-PCR analysis; similarly, six DEGs between FB and NL and 11 DEGs also showed good agreement between the RNA-seq data and qRT-PCR analysis (Fig. 10; Additional file 3: Table S3).

\section{Discussion}

\section{Early flowering of alfalfa is related to hormone} metabolism

Hormones play distinctive roles in controlling plant growth and development, especially in flowering processes [24, 25]. For instance, disruption of auxin a $\quad$ b

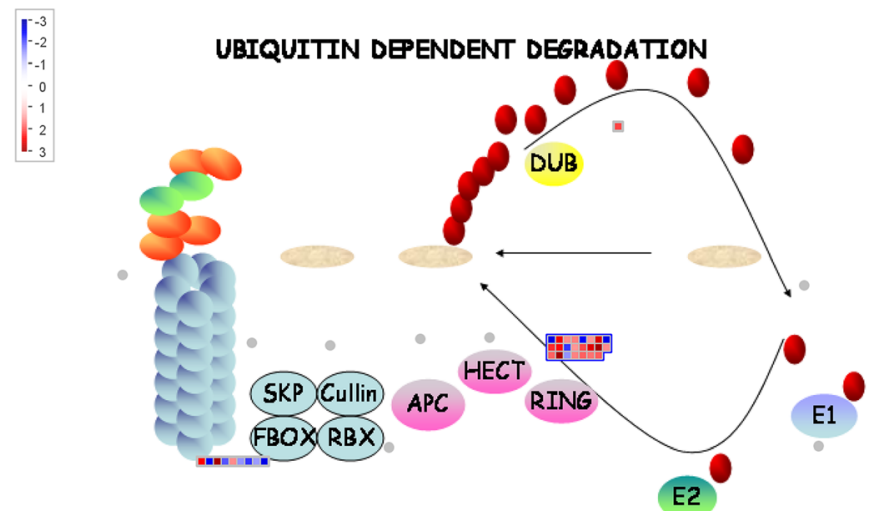

\section{b}

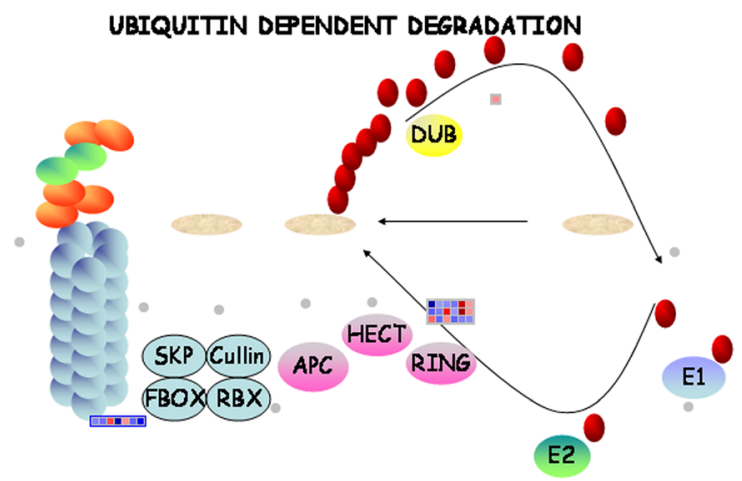

Fig. 8 MapMan display of coordinated changes in the expression levels of genes involved in the ubiquitin-dependent degradation pathway in genotypes 195 and 80 upon the flowering of genotype 80. Shown are DEGs between NL and ML in genotypes 195 (a) and 80 (b). Squares represent DEGs; red and blue indicate up- and downregulated genes, respectively 


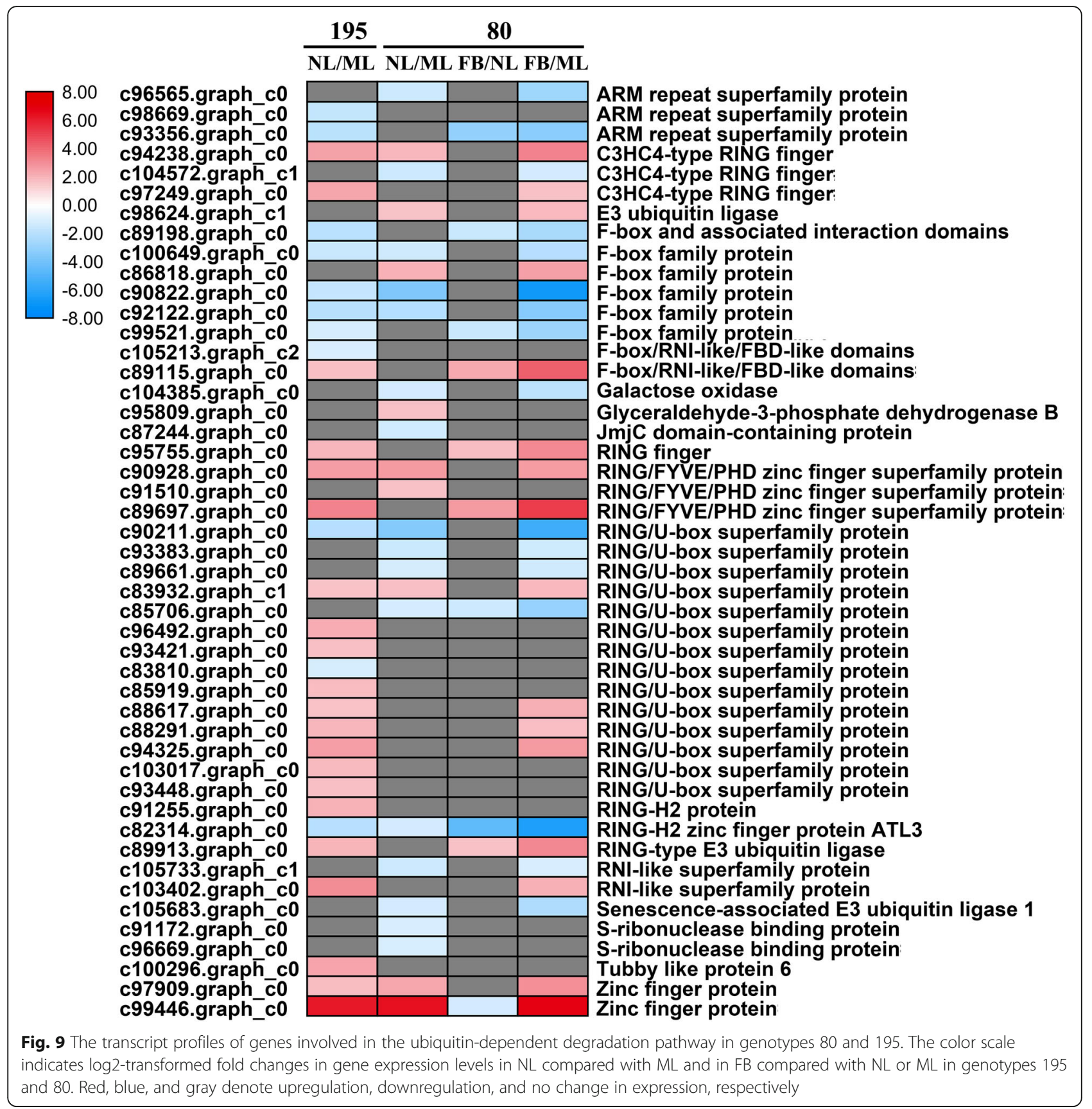

biosynthesis, signaling, or polar auxin transport can extensively inhibit the initiation of inflorescence $[15,26]$ whereas exogenous ABA or JA applications can significantly delay floral transition [13, 14]. Exogenous SA is known to induce flowering by suppressing FLC expression and increasing FT, CO, and SOC1 expression in tobacco (Nicotiana tabacum L.) [27], Lemna paucicostata [28] and Arabidopsis [29]. In the present study, the ratio of hormone contents (IAA, ABA, SA, and JA) in new leaves compared with mature leaves was lower in the early-flowering genotype 80 compared with the late- flowering genotype 195. Consistent with this observation, 11 auxin-related genes were downregulated in NL compared with ML in phenotype 80, these include those encoding SAUR family proteins, o-fucosyltransferase, auxin efflux carrier component 5 , and thromboxane-A synthase. Seven JA-related genes were downregulated in NL compared with ML in genotype 80 , including those encoding the lipoxygenase, the 12-oxophytodienoate reductase-like protein, and SAMT. Meanwhile, we observed the upregulation of genes involved in JA biosynthesis in the FB of genotype 80 , such as genes encoding 


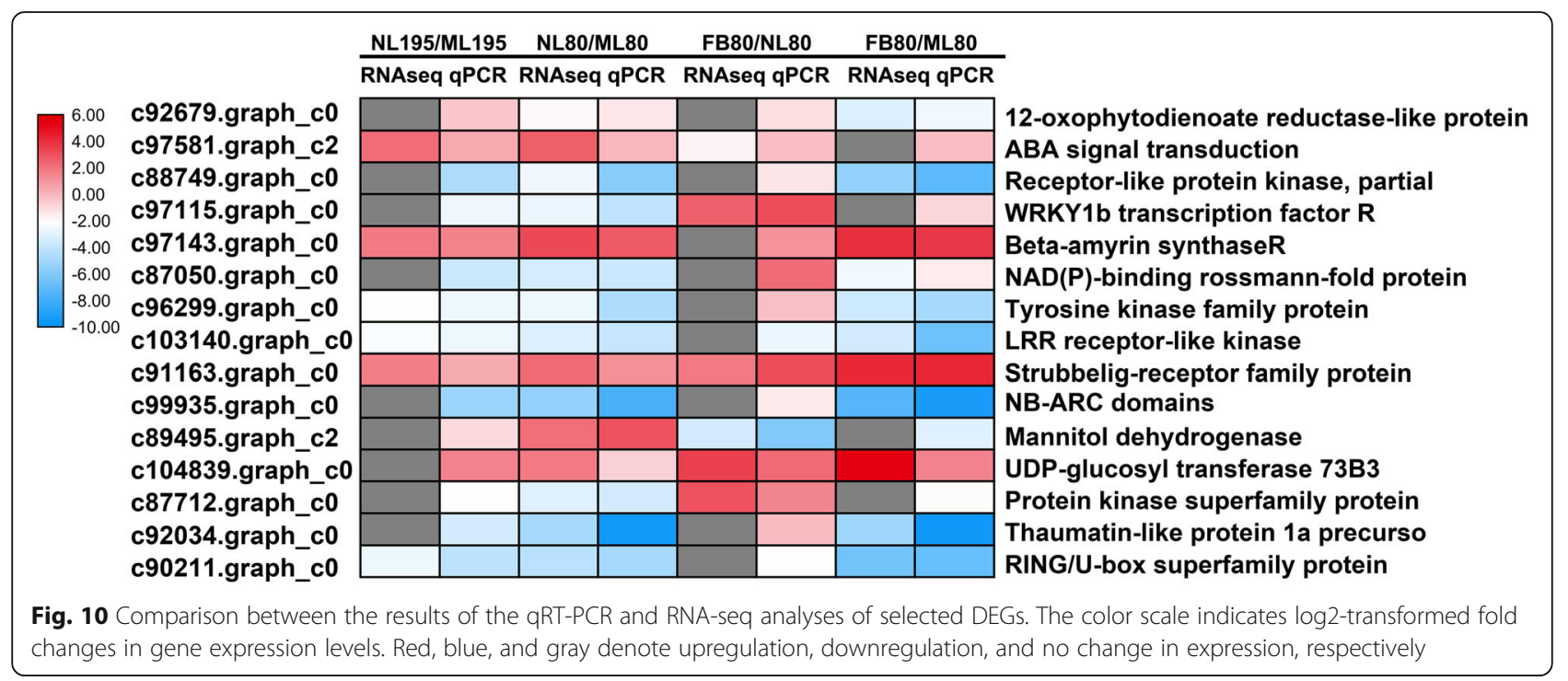

SAMT and lipoxygenase3/5. In addition, we detected higher ABA and SA contents as well as higher transcript levels of the HVA22 family protein and SAMT encoding genes in the $\mathrm{FB}$ of genotype 80 compared with genotype 195. ABA is known to induce AtHAV22 expression mainly in flower buds and inflorescence tissues, whereas SAMT catalyzes the production of methyl-salicylate from SA, which confers pathogen resistance in Arabidopsis and white tea (Camellia sinensis L.) [30-32]. Therefore, the early-flowering phenotype of genotype 80 might be negatively regulated by JA biosynthetic genes, and the interactive networks of these hormones may also contribute to early flowering in alfalfa.

The early flowering of alfalfa is associated with pathogenesis-related and signaling receptor kinase genes Plants often respond to biotic and abiotic stress by diverting more resources away from growth and development to promote early flowering [9]. For instance, Arabidopsis plants infected by fungal and bacterial pathogens showed an accelerated flowering time and increased transcript levels of floral integrator genes $F T$ and GIGANTEA. Further investigation indicated that 19 genes encoding receptor-like proteins were upregulated in the Arabidopsis-Fusarium oxysporum-resistant mutants $[9,33]$. In the present study, $97 \%$ of PR genes were downregulated in the new leaves of genotype 80 , and the genes encoding 11 LRR family proteins, seven receptorlike proteins, and 18 TIR-NBS-LRR class proteins were significantly downregulated. In addition, $67.9 \%$ of the genes involved in the kinase signaling pathway were downregulated in genotype 80 compared with genotype 195, including 38 of the 74 LRR proteins, 12 of the 15 DUF26 kinases, 30 wheat LRK10-like kinases, and $16 \mathrm{~S}-$ locus glycoprotein-like kinases. Previous studies have demonstrated that the overexpression of TaBRI1 (BRASSINOSTEROID-INSENSITIVE1) and ERECTA lead to early flowering and increased slique size in Arabidopsis and tomato, respectively, and overexpression of the tomato LRR receptor-like kinase enhances drought tolerance [34, 35]. In addition, overexpression of AtLRK10L1.2, the homolog of wheat LRK10-like kinase, confers positive response toward ABA signaling and drought tolerance, features associated with early flowering in Arabidopsis [16]. In line with these published results, we found that genotype 80 suffered from pathogen attacks and abiotic stress while exhibiting early flowering during our field experiments. Consistently, pathogenesisrelated and signaling receptor kinase genes were significantly downregulated in genotype 80 compared with the later-flowering genotype 195.

\section{The early flowering of alfalfa is related to secondary metabolism and protein degradation}

2-oxoglutarate/Fe (II)-dependent dioxygenases play versatile roles in secondary metabolite synthesis and catalyze epimerization and demethylation during floral development [36, 37]. For example, PKDM78 and JMJ14, which $2 \mathrm{OG}$ oxygenases, suppress the expression of floral integrator genes $F T, A P 1, S O C 1$, and $L F Y$ by mediating H3K4 demethylation of chromatin, thereby inhibiting floral transition in Arabidopsis [38, 39]. In this study, eight of ten $2 \mathrm{OG}$ oxygenases were significantly downregulated in the new leaves of genotype 80 , indicating that 2OG oxygenases might negatively affect early-flowering. Furthermore, a previous study reported that glycosyltransferases catalyze the glycosylation of plant secondary compounds to maintain cell homeostasis and regulate plant growth and development [40]. Ectopic overexpression of glycosyltransferase genes UGT87A2 and PtGT1 
were found to inhibit $F L C$ expression and increase floral integrator gene expression, thus resulting in an earlyflowering phenotype in Arabidopsis and tobacco, respectively [40, 41]. Here, seven UDP-glycosyltransferases were significantly upregulated in the new leaves of genotype 80 compared with those of genotype 195; these genes were also significantly upregulated in the flower buds compared with mature or new leaves in genotype 80. Together, these results indicate the UGT genes might play critical roles in regulating early flowering in genotype 80 . The widely existed ubiquitin/proteasome system affects plant development by participating in signaling transduction cascades, pathogen defense, and biotic and abiotic stress response [19]. For instance, HOS1 (high expression of osmotically responsive genes1), a RING finger-containing E3 ubiquitin ligase, negatively regulates CONSTANS abundance and delays flowering time in Arabidopsis [42]. By contrast, the FKF1 F-box protein physically interacts with CDF1 (CYCLING DOF FACTOR 1) to induce its degradation, thereby increasing the expression of $\mathrm{CO}$, which promotes earlyflowering in Arabidopsis [43]. In addition, the F-box protein UFO (UNUSUAL FLORAL ORGANS) associates with the AP3 promoter to enhance $L F Y$ transcription [44]. Consistent with these published data, we found that nine of the 11 RING/U-box superfamily proteins were significantly upregulated in new leaves in genotype 195 compared with genotype 80 , indicating that these ubiquitin/proteasome-related genes may be negative regulators of flowering time. Six of seven F-box family proteins were significantly downregulated in the new leaves of genotype 195 compared with genotype 195, suggesting that these F-box proteins may promote early flowering in alfalfa. These findings suggest that secondary metabolism genes and protein degradation genes may be essential for flowering time regulation in alfalfa.

\section{Conclusions}

Based on the phenotypical, physiological and transcriptomic analyses, reduced JA content in new leaves and the downregulation of JA biosynthetic genes, including those encoding lipoxygenases, 12-oxophytodienoate reductase-like proteins, and SAMT may play essential roles in controlling the early-flowering phenotypes in alfalfa. Further analyses reveal that $P R$ genes (including those encoding LRR family proteins, receptor-like proteins, and TIR-NBS-LRR class proteins), genes encoding signaling receptor kinase family members (including DUF26 and wheat LRK10-like kinases), secondary metabolism genes that encode 2OG oxygenases and UGTs, and proteasome degradation pathway genes (such as those encoding RING/U-box superfamily proteins and F-box family proteins) also contribute to the establishment of early-flowering in alfalfa (Fig. 11). In summary, our results provide insights into the regulatory mechanisms underlying early flowering in alfalfa and offer new target genes for future functional characterization and the genetic improvement of alfalfa.

\section{Methods}

Plant materials and growth conditions

Alfalfa genotype 80 (Accession No. PI183404, collected in India) and genotype 195 (Accession No. PI584512, collected in Canada) were provided by the USDA National Plant Germplasm System as previously described [45]. Seedlings were transplanted to the Shangzhuang experimental station of China Agricultural University, Beijing $\left(116.32^{\circ} \mathrm{E}, 40.14^{\circ} \mathrm{N}\right)$ in 2018 . Flowering time investigations for two consecutive years showed that genotype 80 began to flower 20 days earlier than genotype 195 in 2018 and 2019. We propagated their clonal lines from cuttings and grew them in a controlled climate growth chamber at $25^{\circ} / 23^{\circ} \mathrm{C}$ (day/night), with a 16 -h photoperiod, $650 \mathrm{mmol} \mathrm{m}^{-2} \mathrm{~s}^{-1}$ light intensity, and $60 \%$ relative humidity. Three replicates were arranged in a randomized complete block design, each replicate contained three plants (from the same clone of the unanimous genotype) grown in individual pots.

\section{Total RNA extraction, RNA-Seq library construction and sequencing}

When genotype 80 first flowered, mature leaves, new leaves (including apical meristem), and flower buds were collected from three individual plants in each replicate for both genotypes 80 and 195. The harvested tissues were immediately frozen in liquid nitrogen and stored at $-80^{\circ} \mathrm{C}$ for RNA extraction. Total RNA was extracted using the Trizol reagent (Invitrogen, USA) according to the manufacturer's instructions. After DNase treatment, the quality and concentration of the RNA were determined using the Agilent Bioanalyzer 2100 system (Agilent Technologies, USA), samples with a 260:280 ratio of $\geq 2.0$ and RNA integrity number (RIN) of $\geq 8$ were subjected to transcriptome sequencing. RNA libraries were generated using the NEBNext ${ }^{\circ} \mathrm{Ultra}^{\mathrm{mt}}$ RNA Library Prep Kit for Illumina ${ }^{\circ}$ (NEB, USA) following the manufacturer's recommendations. The libraries were sequenced on an Illumina Hiseq 2000 platform (Illumina, USA) to generate paired-end reads. For quality control, raw reads of the FASTQ format were processed through in-house Perl scripts, after which reads containing adapter sequences were removed and $100.13 \mathrm{~Gb}$ of clean reads for fifteen alfalfa samples were obtained. Next, the Q30, GC-content, and sequence duplication level of the clean reads were calculated. All downstream analyses were performed on the clean reads with high quality. The de novo assembly of non-redundant unigenes was performed by using the trinity program (v20131110) [46]. 


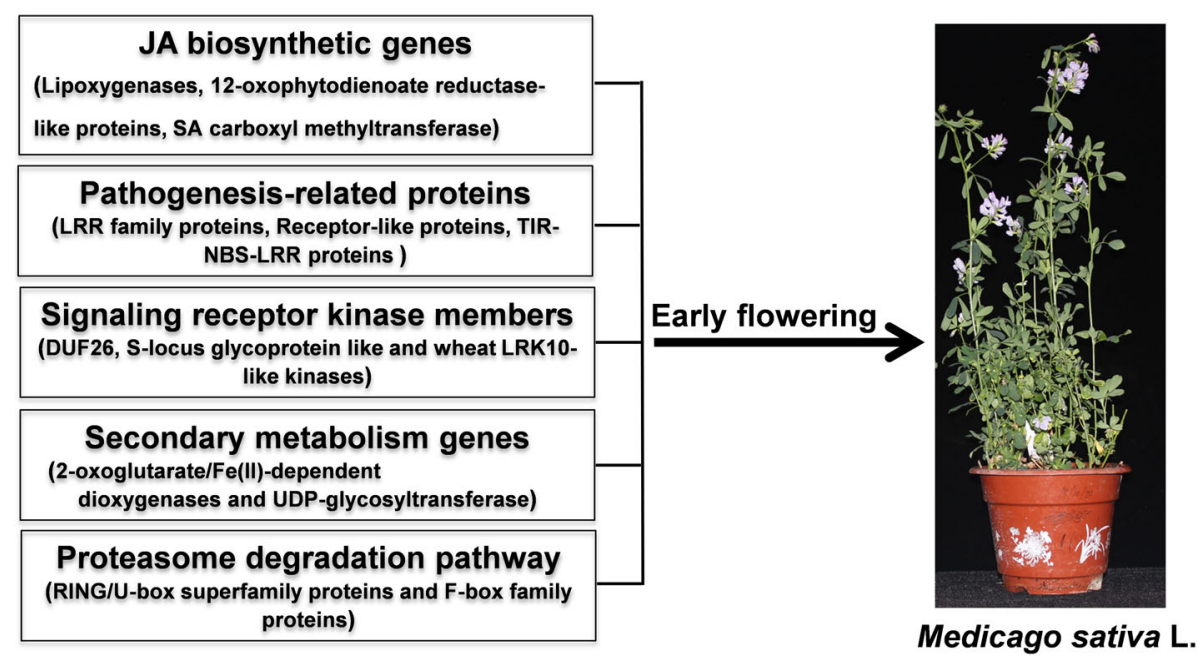

Fig. 11 Diagram of a proposed regulatory network for early flowering in alfalfa

Functional annotation of unigenes and analysis of differentially expressed genes

Functional annotation of the unigenes was performed by BLAST search against public databases, including NR, NT, protein family (Pfam), COG, Swiss-Prot, GO, and KEGG considering E-value threshold $\leq 10^{-5}$. The identification of DEGs between different tissues was performed using the DESeq R package (v1.10.1) [47], and genes with a $\mid \log 2$ fold change $\mid>1.5$ and an adjusted $P$ value $<0.05$ were considered as differentially expressed. For the classification and functional analysis of identified proteins, their homologs were identified using the Phytozome Medicago truncatula genome database (https://phytozome.jgi.doe.gov/pz/portal.html\#!info?alias= Org_Mtruncatula, Mt4.0v1, E-value $<10^{-5}$ ), and the MapMan software (https://mapman.gabipd.org/download, V3.5.1R2) was used for flowering-time associated regulatory network analysis. The Venn graph and heat maps were drawn using TBtools (https://github.com/CJ-Chen/ TBtools/releases).

\section{Endogenous ABA, IAA, JA, and SA analyses in alfalfa}

Endogenous hormone contents were determined in the leaves (the same leaves as those used for the RNA-seq analysis) of genotypes 80 and 195. ABA, IAA, JA, and SA were extracted and purified using previously reported methods [45, 48]. Briefly, $\sim 50 \mathrm{mg}$ of frozen tissue was ground in liquid nitrogen and mixed with $500 \mu \mathrm{L}$ of indolepropionic acid extraction buffer (2:1 dilution) and $50 \mu \mathrm{L}$ of internal standards $\left({ }^{2} \mathrm{H}_{6}\right.$ ABA, ${ }^{2} \mathrm{H}_{2}$ IAA, ${ }^{2} \mathrm{H}_{5} \mathrm{JA}$, and ${ }^{2} \mathrm{H}_{4}$ $\mathrm{SA})$. The mixture was centrifuged at $15000 \mathrm{~g}$ for $5 \mathrm{~min}$, the supernatant was mixed with $1 \mathrm{~mL}$ chloroform, and dissolved in $100 \mu \mathrm{L}$ methanol and used for liquid chromatography coupled with tandem mass spectrometry (LCMS) according to Cao et al. [48] . The content of each hormone was calculated according to the standard curve of the internal standards and normalized to fresh weight (FW) associated with three biological replicates.

\section{qRT-PCR validation of sixteen DEGs}

Total RNA was isolated from the same samples used for the RNA-seq analysis using an RNAprep Pure Plant Kit (Tiangen, Beijing, China) following the manufacturer's instructions. First-stand cDNA was synthesized using the Takara MLV-Reverse transcriptase (Takara Bio, Inc., Otsu, Japan) used for qRT-PCR analysis on the Bio-Rad CFX96 real-time PCR detection system with four biological replicates and using the SYBR Premix Ex Taq (Takara Bio, Inc., Otsu, Japan). Sixteen unigenes were randomly selected for the qRT-PCR analysis using gene-specific qRTPCR primers (Additional file 3: Table S3). The $2^{-\Delta \Delta C T}$ method [49] was used to calculate the relative expression level of each gene to the endogenous control Actin.

\section{Statistical analysis}

All data were subjected to the analysis of variance (ANOVA) in SAS 9.0 (SAS Institute, Cary, NC) using the general linear model. Fisher's least significant difference test (LSD, $P<0.05$ was used to determine significant differences among treatments according to Ma et al. [50].

\section{Supplementary Information}

The online version contains supplementary material available at https://doi. org/10.1186/s12870-020-02775-9.

Additional file 1: Table S1. Annotation of DEGs identified in genotypes 195 and 80.

Additional file 2: Table S2. Mapman display of the functional categories of DEGs in genotypes 195 and 80. 
Additional file 3: Table S3. $q R T-P C R$ validation of selected DEGs identified by RNA-seq in genotypes 195 and 80 .

\section{Abbreviations}

FT: FLOWERING LOCUS T; FLC: FLOWERING LOCUS C; CO: CONSTANS: SOC1: SUPPRESSOR OF OVEREXPRESSION OF CONSTANS1; SPL: SQUAMOSA PROMOTER BINDING-LIKE; JA: Jasmonate-activated; ABA: Abscisic acid; ABI5: ABSCISIC ACID-INSENSITIVE MUTANT 5; RLK: Receptor-like kinase; LRR: Leucine-rich repeat; PR: Pathogenesis-related; IAA: Auxin; SA: Salicylic acid; SAMT: SA carboxyl methyltransferase; TIR-NBS-LRR: Toll-interleukin-like receptor -nucleotide-binding site-LRR; CC-NBS-LRR: Coiled-coil-NBS-LRR; NBARC: Nucleotide-binding adaptor shared by APAF-1, R proteins, and CED-4; 2OG oxygenases: 2-oxoglutarate and Fe (II)-dependent oxygenase superfamily proteins; BRI1: BRASSINOSTEROID-INSENSITIVE1; CDF1: CYCLING DOF FACTOR 1; UFO: UNUSUAL FLORAL ORGANS; UGTs: UDPGlycosyltransferase superfamily proteins; DUF 26: Domain of unknown function 26

\section{Acknowledgements}

We thank A \& L Scientific Editing (www.alpublish.com) for its linguistic assistance during the preparation of this manuscript.

\section{Authors' contributions}

$\mathrm{DM}, \mathrm{BL}$, and $\mathrm{XM}$ designed and performed the experiments; $\mathrm{DM}, \mathrm{BL}$, and $\mathrm{XM}$ wrote the manuscript; XM and PM conceived the study, supervised the project, and edited the manuscript. LG, YW, XC, and FL assisted in performing the experiments. All authors read and approved the final manuscript.

\section{Funding}

This research was supported by the National natural science foundation of China (31760698), the Ningxia agricultural breeding project (2019NYYZ0401), the key research and development project of Ningxia (2019bbf02022-042), the first-class discipline construction project of Ningxia University (Ecology, nxylxk2017b06), and the Fundamental Research Funds for the Central Universities (2018QC144). Founding body had no role in the design of the study and collection, analysis, and interpretation of data and in writing the manuscript.

\section{Availability of data and materials}

The RNA-seq data have been deposited into NCBI under accession number PRJNA602419 (https://dataview.ncbi.nlm.nih.gov/object/PRJNA602419).

\section{Ethics approval and consent to participate}

Not applicable.

\section{Consent for publication}

Not applicable.

\section{Competing interests}

The authors declare that they have no competing interests.

\section{Author details}

${ }^{1}$ Breeding Base for State Key Laboratory of Land Degradation and Ecological Restoration in Northwest China/ Ministry of Education Key Laboratory for Restoration and Reconstruction of Degraded Ecosystems in Northwest China, Ningxia University, Yinchuan 750021, China. ${ }^{2}$ College of Grassland Science and Technology, China Agricultural University, Beijing 100193, China. ${ }^{3}$ National Animal Husbandry Service, Maizidian Street, North Nongzhan Road, Chaoyang District, Beijing 100125, China.

Received: 31 August 2020 Accepted: 2 December 2020

Published online: 06 January 2021

\section{References}

1. Singer SD, Hannoufa A, Acharya S. Molecular improvement of alfalfa for enhanced productivity and adaptability in a changing environment. Plant Cell Environ. 2018;41(9):1955-71.

2. Kumar S. Biotechnological advancements in alfalfa improvement. J Apple Genet. 2011;52(2):111-24
3. Adhikari L, Makaju SO, Missaoui AM. QTL mapping of flowering time and biomass yield in tetraploid alfalfa (Medicago sativa L.). BMC Plant Biol. 2019; 19(1):359

4. Fan W, Ge G, Liu Y, Wang W, Liu L, Jia Y. Proteomics integrated with metabolomics: analysis of the internal causes of nutrient changes in alfalfa at different growth stages. BMC Plant Biol. 2018;18(1):78.

5. Andrés F, Coupland $\mathrm{G}$. The genetic basis of flowering responses to seasonal cues. Nat Rev Genet. 2012;13(9):627-39.

6. Blümel $M$, Dally $N$, Jung C. Flowering time regulation in crops-what did we learn from Arabidopsis? Curr Opin Biotech. 2015;32:121-9.

7. Fornara F, de Montaigu A, Coupland G. SnapShot: control of flowering in Arabidopsis. Cell. 2010;141(3):550.

8. Kang J, Zhang T, Guo T, Ding W, Long R, Yang Q, Wang Z. Isolation and Functional Characterization of MsFTa, a FLOWERING LOCUS T Homolog from Alfalfa (Medicago sativa L.). Int J Mol Sci. 2019;20(8):1968.

9. Lyons R, Rusu A, Stiller J, Powell J, Manners JM, Kazan K. Investigating the association between flowering time and defense in the Arabidopsis thaliana-Fusarium oxysporum interaction. PLoS One. 2015;10(6):e0127699.

10. Wang G, Köhler C. Epigenetic processes in flowering plant reproduction. J Exp Bot. 2017;68(4):797-807.

11. Wang JW. Regulation of flowering time by the miR156-mediated age pathway. J Exp Bot. 2014;65(17):4723-30.

12. Liu F, Quesada V, Crevillén P, Bäurle I, Swiezewski S, Dean C. The Arabidopsis RNA-binding protein FCA requires a lysine-specific Demethylase 1 homolog to Downregulate FLC. Mol Cell. 2007;28(3):398-407.

13. Wang H, Li Y, Pan J, Lou D, Hu Y, Yu D. The bHLH transcription factors MYC2, MYC3, and MYC4 are required for Jasmonate-mediated inhibition of flowering in Arabidopsis. Mol Plant. 2017;10(11):1461-4.

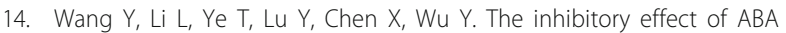
on floral transition is mediated by ABI5 in Arabidopsis. J Exp Bot. 2013; 64(2):675-84

15. Campos-Rivero G, Osorio-Montalvo P, Sánchez-Borges R, Us-Camas R, Duarte-Aké F, De-la-Peña C. Plant hormone signaling in flowering: an epigenetic point of view. J Plant Physiol. 2017:214:16-27.

16. Lim CW, Yang SH, Shin KH, Lee SC, Kim SH. The AtLRK10L1.2, Arabidopsis ortholog of wheat LRK10, is involved in ABA-mediated signaling and drought resistance. Plant Cell Rep. 2015;34(3):447-55.

17. Torti S, Fornara F, Vincent C, Andrés F, Nordström K, Göbel U, Knoll D, Schoof H, Coupland G. Analysis of the Arabidopsis shoot meristem transcriptome during floral transition identifies distinct regulatory patterns and a leucine-rich repeat protein that promotes flowering. Plant Cell. 2012; 24(2):444-62.

18. Gachomo EW, Jno Baptiste L, Kefela T, Saidel WM, Kotchoni SO. The Arabidopsis CURVY1 (CVY1) gene encoding a novel receptor-like protein kinase regulates cell morphogenesis, flowering time and seed production. BMC Plant Biol. 2014;14(1):221.

19. Sharma M. Role of Plant U-BOX (PUB) Protein in Stress and Development Plant Stress. 2013;7:1-9.

20. Li W, Dai L, Wang GL. PUB13, a U-box/ARM E3 ligase, regulates plant defense, cell death, and flowering time. Plant Signal Behav. 2012;7(8): 898-900.

21. Sun H, Long R, Zhang F, Zhang T, Kang J, Wang Z, Cao C, Yu J, Yang Q. Proteomic analysis of shoot tips from two alfalfa cultivars with different florescence. Plant Mol Biol Rep. 2019;37(4):265-76.

22. Thomson B, Wellmer F. Molecular regulation of flower development. Curr Top Dev Biol. 2019;131:185-210.

23. ÓMaoiléidigh DS, Wuest SE, Rae L, Raganelli A, Ryan PT, Kwasniewska K, Das P, Lohan AJ, Loftus B, Graciet E, Wellmer F. Control of reproductive floral organ identity specification in Arabidopsis by the $C$ function regulator AGAMOUS. Plant Cell. 2013:25(7):2482-503.

24. Cho LH, Yoon J, An G. The control of flowering time by environmental factors. Plant J. 2017:90(4):708-19.

25. Davis SJ. Integrating hormones into the floral-transition pathway of Arabidopsis thaliana. Plant Cell Environ. 2009:32(9):1201-10.

26. Cheng Y, Zhao Y. A role for Auxin in flower development. J Integr Plant Biol. 2007:49(1):99-104.

27. Lee TT, Skoog F. Effects of substituted phenols on bud formation and growth of tobacco tissue cultures. Physiol Plant. 1965;18(2):386-402.

28. Khurana JP, Cleland CF. Role of salicylic acid and benzoic acid in flowering of a photoperiod-Insensitive strain, Lemna paucicostata LP6. Plant Physiol. 1992;100(3):1541-6. 
29. Martínez C, Pons E, Prats G, León J. Salicylic acid regulates flowering time and links defence responses and reproductive development. Plant J. 2004; 37(2):209-17.

30. Chen CN, Chu CC, Zentella R, Pan SM, Ho TH. AtHVA22 gene family in Arabidopsis: phylogenetic relationship, $\mathrm{ABA}$ and stress regulation, and tissue-specific expression. Plant Mol Biol. 2002;49(6):633-44.

31. Koo YJ, Kim MA, Kim EH, Song JT, Jung C, Moon JK, Kim JH, Seo HS, Song SI, Kim JK, Lee JS, Cheong JJ, Choi YD. Overexpression of salicylic acid carboxyl methyltransferase reduces salicylic acid-mediated pathogen resistance in Arabidopsis thaliana. Plant Mol Biol. 2007;64(1-2):1-15.

32. Deng WW, Wang R, Yang T, Ln J, Zhang ZZ. Functional characterization of salicylic acid carboxyl methyltransferase from Camellia sinensis, providing the aroma compound of methyl salicylate during the withering process of white tea. J Agric Food Chem. 2017;65(50):11036-45.

33. Korves TM, Bergelson J. A developmental response to pathogen infection in Arabidopsis. Plant Physiol. 2003;133(1):339-47.

34. Singh A, Breja P, Khurana JP, Khurana P. Wheat Brassinosteroid-Insensitive (TaBRI1) interacts with members of TaSERK gene family and cause early flowering and seed yield enhancement in Arabidopsis. PLoS One. 2016; 11(6):e0153273.

35. Villagarcia H, Morin AC, Shpak ED, Khodakovskaya MV. Modification of tomato growth by expression of truncated ERECTA protein from Arabidopsis thaliana. J Exp Bot. 2012;63(18):6493-504.

36. Jia B, Jia X, Kim KH, Jeon CO. Integrative view of 2-oxoglutarate/Fe (II)dependent oxygenase diversity and functions in bacteria. Biochim Biophys Acta Gen Subj. 2017:1861(2):323-34

37. Farrow SC, Facchini PJ. Functional diversity of 2-oxoglutarate/Fe (II)dependent dioxygenases in plant metabolism. Front Plant Sci. 2014;5:524.

38. Lu F, Cui X, Zhang S, Liu C, Cao X. JMJ14 is an H3K4 demethylase regulating flowering time in Arabidopsis. Cell Res. 2010;20(3):387-90,

39. Yang W, Jiang D, Jiang J, He Y. A plant-specific histone H3 lysine 4 demethylase represses the floral transition in Arabidopsis. Plant J. 2010;62(4): 663-73.

40. Wang YW, Wang WC, Jin SH, Wang J, Wang B, Hou BK. Over-expression of a putative poplar glycosyltransferase gene, PtGT1, in tobacco increases lignin content and causes early flowering. J Exp Bot. 2012;63(7):2799-808.

41. Wang B, Jin $\mathrm{SH}$, Hu HQ, Sun YG, Wang YW, Han P, Hou BK. UGT87A2, an Arabidopsis glycosyltransferase, regulates flowering time via FLOWERING LOCUS C. New Phytol. 2012;194(3):666-75.

42. Lazaro A, Valverde F, Piñeiro M, Jarillo JA. The Arabidopsis E3 ubiquitin ligase HOS1 negatively regulates CONSTANS abundance in the photoperiodic control of flowering. Plant Cell. 2012;24(3):982-99.

43. Imaizumi T, Schultz TF, Harmon FG, Ho LA, Kay SA. FKF1 F-box protein mediates cyclic degradation of a repressor of CONSTANS in Arabidopsis. Science. 2005;309(5732):293-7.

44. Chae E, Tan QK, Hill TA, Irish VF. An Arabidopsis F-box protein acts as a transcriptional co-factor to regulate floral development. Development. 2008; 135(7):1235-45.

45. Ge L, Zhang K, Cao X, Weng Y, Liu B, Mao P, Ma X. Sequence characteristics of Medicago truncatula cyclophilin family members and function analysis of MsCYP20-3B involved in axillary shoot development. Mol Biol Rep. 2020; 47(2):907-19.

46. Grabherr MG, Haas BJ, Yassour M, Levin JZ, Thompson DA, Amit I, Adiconis $X$, Fan $L$, Raychowdhury R, Zeng Q. Full-length transcriptome assembly from RNA-Seq data without a reference genome. Nat Biotechnol. 2011;29(7):644.

47. Anders S, Huber W. Differential expression of RNA-Seq data at the gene level-the DESeq package. Heidelberg: European Molecular Biology Laboratory (EMBL); 2012

48. Cao J, Li M, Chen J, Liu P, Li Z. Effects of MeJA on Arabidopsis metabolome under endogenous JA deficiency. Sci Rep. 2016;6:37674.

49. Livak KJ, Schmittgen TD. Analysis of relative gene expression data using real-time quantitative $P C R$ and the 2(-Delta Delta $C(T))$ method. Methods. 2001;25(4):402-8.

50. Ma X, Xu Q, Meyer WA, Huang B. Hormone regulation of rhizome development in tall fescue (Festuca arundinacea) associated with proteomic changes controlling respiratory and amino acid metabolism. Ann Bot. 2016; 118(3):481-94.

\section{Publisher's Note}

Springer Nature remains neutral with regard to jurisdictional claims in published maps and institutional affiliations.

\section{Ready to submit your research? Choose BMC and benefit from:}

- fast, convenient online submission

- thorough peer review by experienced researchers in your field

- rapid publication on acceptance

- support for research data, including large and complex data types

- gold Open Access which fosters wider collaboration and increased citations

- maximum visibility for your research: over $100 \mathrm{M}$ website views per year

At BMC, research is always in progress.

Learn more biomedcentral.com/submissions 